\title{
Scalings and Limits of Landau-de Gennes Models for Liquid Crystals: A Comment on Some Recent Analytical Papers*
}

\author{
Eugene C. Gartland, Jr. \\ Kent State University \\ Department of Mathematical Sciences, Kent, Ohio 44242, USA \\ E-mail(corresp.): gartland@math.kent.edu
}

Received August 28, 2017; revised May 11, 2018; accepted May 14, 2018

\begin{abstract}
Some recent analytical papers have explored limiting behaviors of Landaude Gennes models for liquid crystals in certain extreme ranges of the model parameters: limits of "vanishing elasticity" (in the language of some of these papers) and "low-temperature limits." We use simple scaling analysis to show that these limits are properly interpreted as limits in which geometric length scales (such as the size of the domain containing the liquid crystal material) become large compared to intrinsic length scales (such as correlation lengths or coherence lengths, which determine defect core sizes). This represents the natural passage from a mesoscopic model to a macroscopic model and is analogous to a "London limit" in the Ginzburg-Landau theory of superconductivity or a "large-body limit" in the Landau-Lifshitz theory of ferromagnetism. Known relevant length scales in these parameter regimes (nematic correlation length, biaxial coherence length) can be seen to emerge via balances in equilibrium Euler-Lagrange equations associated with well-scaled Landau-de Gennes free-energy functionals.
\end{abstract}

Keywords: liquid crystals, Landau-de Gennes model, Oseen-Frank model, large-body limit, low-temperature limit.

AMS Subject Classification: 82D30; 00A73; 35B40.

\section{Introduction}

The Landau-de Gennes and Oseen-Frank models are the two most widely used continuum models to characterize equilibrium orientational properties of materials in the nematic liquid crystal phase. The Landau-de Gennes model is

\footnotetext{
* Supported in part by U.S. National Science Foundation grant DMS-1211597

Copyright (c) 2018 The Author(s). Published by VGTU Press

This is an Open Access article distributed under the terms of the Creative Commons Attribution License (http://creativecommons.org/licenses/by/4.0/), which permits unrestricted use, distribution, and reproduction in any medium, provided the original author and source are credited.
} 
a mesoscopic phenomenological model expressed in terms of an integral functional of a tensor field $\mathbf{Q}$, the "tensor order parameter." In its simplest form (the "equal elastic constant model"), the functional can be written

$$
\mathcal{F}[\mathbf{Q}]=\int_{\Omega}\left[\frac{L}{2}|\nabla \mathbf{Q}|^{2}+f_{\mathrm{b}}(\mathbf{Q})\right] \mathrm{d} V .
$$

Here $\mathcal{F}$ gives the free energy of a material occupying the region $\Omega$, and $f_{\mathrm{b}}$ represents the free energy per unit volume of a homogeneous bulk material, which in most cases is given in the form

$$
f_{\mathrm{b}}(\mathbf{Q})=\frac{A}{2} \operatorname{tr}\left(\mathbf{Q}^{2}\right)-\frac{B}{3} \operatorname{tr}\left(\mathbf{Q}^{3}\right)+\frac{C}{4} \operatorname{tr}\left(\mathbf{Q}^{2}\right)^{2} .
$$

The parameters $L, A, B$, and $C$ are material dependent, with $L, B$, and $C$ positive and $A$ (which can be positive, negative, or zero) usually taken to have a simple linear dependence on temperature:

$$
A=a_{0}\left(T-T_{\mathrm{SC}}\right) .
$$

Here $a_{0}$ is a positive constant, $T$ denotes temperature, and $T_{\mathrm{SC}}$ represents the "super-cooling temperature" (sometimes denoted $T^{*}$ ), which is discussed below. Stationary points of $\mathcal{F}$ give equilibrium orientational states, with the global minimum determining the structural phase of the system for a given set of boundary conditions and parameter values.

The $\mathbf{Q}$ tensor is formally defined as the traceless part of the second-moment tensor of the orientational probability distribution function (which, if known, would contain complete information about the orientational state at a point): $\mathbf{Q}=\langle\boldsymbol{l} \otimes \boldsymbol{l}\rangle-\frac{1}{3} \mathbf{I}$. Here the unit vector $\boldsymbol{l}$ denotes the direction of the distinguished axis of the anisometric molecular architecture (usually the long axis of elongated, rod-like molecules), and $\mathbf{I}$ is the identity tensor. As such, $\mathbf{Q}$ is real, symmetric, traceless, and generically would have three distinct real eigenvalues and associated orthogonal eigenvectors, representing a "biaxial" state of order. The eigenvectors of $\mathbf{Q}$ provide information about the directions of orientational ordering at a point, while the eigenvalues give information about the degrees of order-note that the value of $f_{\mathrm{b}}$ depends only on the eigenvalues of $\mathbf{Q}$. An isotropic (totally disordered) state corresponds to $\mathbf{Q}=\mathbf{0}$ (the zero tensor).

For given $B, C>0$, the shape of the surface of $f_{\mathrm{b}}$ as a function of the two independent eigenvalues of $\mathbf{Q}$ changes as temperature changes. For $T>$ $T_{\mathrm{SH}}>T_{\mathrm{SC}}$, where $T_{\mathrm{SH}}$ denotes the "super-heating temperature," $\mathbf{Q}=\mathbf{0}$ is the global minimizer of $f_{\mathrm{b}}$, and no ordered phase exists- $f_{\mathrm{b}}$ has no other stationary points. For $T<T_{\mathrm{SC}}$, the isotropic phase ceases to be locally stable, and the only minimizer of $f_{\mathrm{b}}$ is a $\mathbf{Q}$ tensor with a dominant eigenvalue and a degenerate pair of equal eigenvalues. Since $\operatorname{tr}(\mathbf{Q})=0$, such a $\mathbf{Q}$ can be written

$$
\mathbf{Q}=S(\boldsymbol{n} \otimes \boldsymbol{n}-\mathbf{I} / 3) \text {. }
$$

Here $S$ is referred to as the "scalar order parameter," and $\boldsymbol{n}$ is the distinguished eigenvector. This is known as a "uniaxial" state of order. The terms "uniaxial" and "biaxial" come from the optical properties of materials in these states, 

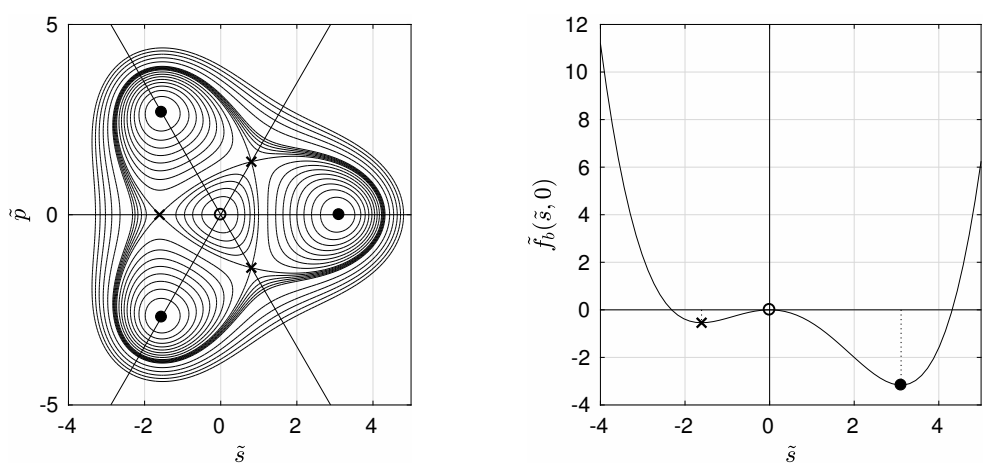

Figure 1. (Left) Contour plot of $\tilde{f}_{\mathrm{b}}(\tilde{s}, \tilde{p})$, a dimensionless form of the bulk free-energy density $f_{\mathrm{b}}$ in (1.1b) with $\mathbf{Q}$ parameterized as in (1.4), using coordinates $\tilde{s}=\sqrt{6} s, \tilde{p}=\sqrt{2} p$. The order tensor $\mathbf{Q}$ is biaxial except along the lines $\tilde{p}=0, \tilde{p}= \pm \sqrt{3} \tilde{s}$ (where it is uniaxial). (Right) Section of the $\tilde{f}_{\mathrm{b}}$ surface cut along the $\tilde{s}$ axis. Critical points: global minima (solid circles), local maximum (open circle), saddles (crosses).

which are the same as those of a uniaxial crystal or a biaxial crystal. For $T$ in the narrow range between $T_{\mathrm{SC}}$ and $T_{\mathrm{SH}}$, both the isotropic and the uniaxial phases are locally stable, and there is a first-order phase transition at the "nematic-isotropic transition temperature" $T_{\mathrm{NI}}$, below which the ordered phase becomes the global minimizer.

A visualization can be helpful to understand $f_{\mathrm{b}}$ as a function of $\mathbf{Q}$. The symmetric traceless tensor $\mathbf{Q}$ is diagonal in its eigenframe $\left(\boldsymbol{e}_{1}, \boldsymbol{e}_{2}, \boldsymbol{e}_{3}\right.$, say), and a simple parameterization of its eigenvalues is given by

$$
\mathbf{Q}=2 s \boldsymbol{e}_{1} \otimes \boldsymbol{e}_{1}+(-s+p) \boldsymbol{e}_{2} \otimes \boldsymbol{e}_{2}+(-s-p) \boldsymbol{e}_{3} \otimes \boldsymbol{e}_{3}
$$

In this representation, the isotropic state corresponds to $s=p=0$, while uniaxial states of the form (1.3) are associated with the cases $p=0\left(\boldsymbol{n}=\boldsymbol{e}_{1}\right.$, $S=3 s), p=-3 s\left(\boldsymbol{n}=\boldsymbol{e}_{2}, S=-6 s\right)$, and $p=3 s\left(\boldsymbol{n}=\boldsymbol{e}_{3}, S=-6 s\right)$. Thus, in the $s-p$ plane, $\mathbf{Q}$ is biaxial everywhere except along three straight lines through the origin. Figure 1 contains a contour plot of $\tilde{f}_{\mathrm{b}}(\tilde{s}, \tilde{p})$, a certain dimensionless version of $f_{\mathrm{b}}$ expressed as a function of the normalized coordinates $\tilde{s}=\sqrt{6} \mathrm{~s}$ and $\tilde{p}=\sqrt{2} p$. In terms of these, $\mathbf{Q}=\tilde{s} \mathbf{E}_{1}+\tilde{p} \mathbf{E}_{2}$ (with symmetric traceless tensors $\mathbf{E}_{1}$ and $\mathbf{E}_{2}$ satisfying $\left.\mathbf{E}_{i} \cdot \mathbf{E}_{j}=\delta_{i j}\right)$, tr $\mathbf{Q}^{2}=\tilde{s}^{2}+\tilde{p}^{2}$, and the uniaxial lines are given by $\tilde{p}=0$ and $\tilde{p}= \pm \sqrt{3} \tilde{s}$. The contour plot corresponds to a temperature $T<T_{\mathrm{SC}}$. The three solid circles give equivalent global minima; the open circle at the origin is a local maximum point; and the three crosses are saddles. The right half of Figure 1 contains a section of the surface cut along $\tilde{p}=0$-the same curve would be obtained along $\tilde{p}= \pm \sqrt{3} \tilde{s}$.

Simple relationships among $A, B$, and $C$ are associated with the temperatures $T_{\mathrm{SC}}, T_{\mathrm{NI}}$, and $T_{\mathrm{SH}}$ :

$$
T_{\mathrm{SC}}<T_{\mathrm{NI}}<T_{\mathrm{SH}} \leftrightarrow A_{\mathrm{SC}}=0<A_{\mathrm{NI}}=\frac{B^{2}}{27 C}<A_{\mathrm{SH}}=\frac{B^{2}}{24 C}
$$

Every material that admits a nematic liquid crystal phase would reside in such a state for some finite range of temperatures below $T_{\mathrm{NI}}$; Landau-de Gennes 
models (which are formally derived via truncated expansions) contain no parameters that indicate the extent of this range, below which actual materials either crystallize or transition to a glass phase or to another liquid crystal phase.

The distortional elasticity term associated with the constant $L$ in (1.1a) penalizes spatial variations of the $\mathbf{Q}$ tensor, while the bulk ordering potential $f_{\mathrm{b}}$ strives to put the eigenvalues of $\mathbf{Q}$ into certain wells. More complete versions of this model can contain additional terms incorporating more elastic constants, more bulk constants, terms associated with couplings to electric or magnetic fields, terms associated with chirality, flexoelectric effects, ferroelectric effects, surface anchoring potentials, and more. See [18] for an introduction to the model and for further references. The form (1.1) is the simplest form of the model and is sufficient for the purposes at hand. Landau-de Gennes models occupy a similar position in the theory of liquid crystals to that of GinzburgLandau models for the theory of superconductivity and Landau-Lifshitz models for ferromagnetism.

The Oseen-Frank model is a macroscopic phenomenological model for liquid crystal orientational properties. It is expressed in terms of an integral functional of a unit-length vector field $\boldsymbol{n}$ (the "director field"). In its simplest ("equal elastic constant") form, the functional can be written

$$
F[\boldsymbol{n}]=\frac{K}{2} \int_{\Omega}|\nabla \boldsymbol{n}|^{2} \mathrm{~d} V .
$$

Here $F$ gives the distortional elastic energy of the material occupying $\Omega$, and $\boldsymbol{n}$ represents the average orientation of the distinguished axes of the molecules in a fluid element at a point (and can be identified with the eigenvector associated with the distinguished eigenvalue of a uniaxial $\mathbf{Q}$ tensor, as in (1.3)). The elastic constant $K$ is a material-dependent and temperature-dependent parameter. Equilibrium orientational states are given by stationary points of $F$ constrained by boundary conditions and the pointwise unit-length constraint on $\boldsymbol{n}$, with the structural phase of the system (for a given $K$ and boundary conditions) again given by the global minimizer. As is the case with the Landau-de Gennes free energy $\mathcal{F}$, more realistic models for $F$ involve many more terms, parameters, couplings, and effects. Standard references include [27,28]. Oseen-Frank models have been widely and successfully used for many years to model liquid crystal systems at the scales of typical devices and experiments.

These two models differ in several ways. The Landau-de Gennes model allows for both spatially varying degrees of order and biaxiality, and it is expressed in terms of a tensor field. The Oseen-Frank model, on the other hand, assumes a uniform degree of orientational order, as well as a uniaxial state of order, and is expressed in terms of a vector field. Another difference between the models is that common liquid crystal "defects" (such as "point defects" and "disclination lines") are singularities of the director field in the Oseen-Frank model, whereas in the Landau-de Gennes model, these have a small but finite "core size" and the associated tensor field Q remains smooth throughout a neighborhood of such a defect. In Oseen-Frank free-energy functionals, such as (1.6), point defects have finite free energy, while the free energy of a disclina- 
tion line is infinite. In the Landau-de Gennes model, however, the free energies of both are finite. In general, defects are caused by the conflicting demands of distortional elasticity, boundary conditions, external electric or magnetic fields, and the like. Landau-de Gennes models are typically employed in problems in which geometric length scales are not too large compared to intrinsic length scales (such as defect core sizes), while Oseen-Frank models are used when the geometric length scale is much larger than the core size (and the fine-detail structure of the core is not important).

In recent years, Landau-de Gennes models have received considerable attention from the mathematical analysis community. In particular, papers have appeared that rigorously explore (among other issues) limiting behaviors of this model for certain extreme ranges of the elastic constants $[2,12,16]$. Analyses of somewhat related limits (emphasizing behavior at low temperatures) are found in $[4,13]$. Our main purpose here is to explain how these limits should be interpreted.

In [16] (the earliest of these papers), the model is taken in the form (1.1) above, and the authors motivate their work as follows: "we study the limit of vanishing elastic constant $L \rightarrow 0 \ldots$ the limit $L \rightarrow 0$ is a physically relevant limit since the elastic constant $L$ is typically very small, of the order $10^{-11} \mathrm{~J} / \mathrm{m}$." In [2], a slightly more general form of the model is used:

$$
\mathcal{F}_{\varepsilon}[\mathbf{Q}]=\int_{\Omega}\left[f_{\mathrm{e}}(\nabla \mathbf{Q})+\varepsilon^{-2} f_{\mathrm{b}}(\mathbf{Q})\right] \mathrm{d} V,
$$

where

$$
f_{\mathrm{e}}(\nabla \mathbf{Q})=\frac{L_{1}}{2} Q_{i j, k} Q_{i j, k}+\frac{L_{2}}{2} Q_{i j, j} Q_{i k, k}+\frac{L_{3}}{2} Q_{i j, k} Q_{i k, j} .
$$

Here $Q_{i j}$ are the components of $\mathbf{Q}$ with respect to a fixed Cartesian frame; $Q_{i j, k}$ denotes $\partial Q_{i j} / \partial x_{k}$; and summation over repeated indices is implied. The dimensionless parameter $\varepsilon$ is artificially introduced in order to be able to drive the elastic constants $L_{1}, L_{2}$, and $L_{3}$ to zero simultaneously. The $L_{1}$ term above corresponds to the $L$ term in (1.1a). The authors motivate their work "Our goal in this paper is to investigate minimizers [of (1.7)] and to analyze their behavior in the vanishing elastic energy limit, $\varepsilon \rightarrow 0$."

In these two papers, the models are analyzed in fully dimensional form. In [12], a dimensionless model is studied, and it is expressed in terms of the second-moment tensor $\mathbf{u}=\langle\boldsymbol{l} \otimes \boldsymbol{l}\rangle$ (instead of $\mathbf{Q}$ ). The model there is written

$$
E_{\varepsilon}[\mathbf{u}]=\int_{\Omega}\left[\frac{1}{2}|\nabla \mathbf{u}|^{2}+\frac{1}{\varepsilon^{2}} W(\mathbf{u})\right] \mathrm{d} V, \quad W(\mathbf{u})=\frac{1}{2} \operatorname{tr}\left(\left(\mathbf{u}-\mathbf{u}^{2}\right)^{2}\right) .
$$

The bulk ordering potential $W$ here is constructed by design to have a minimizer (in the class $\operatorname{tr}(\mathbf{u})=1$ ) at a perfectly ordered uniaxial state $\mathbf{u}=\boldsymbol{n} \otimes \boldsymbol{n}$, with $|\boldsymbol{n}|=1$ but with the direction of $\boldsymbol{n}$ arbitrary, and $\varepsilon$ is referred to as a "dimensionless elastic constant." With the help of the relation $\mathbf{u}=\mathbf{Q}+\frac{1}{3} \mathbf{I}$, the model can be identified with a certain constrained form of (1.1). Again, the limit $\varepsilon \rightarrow 0$ is explored. 
All three of these papers explore similar limits, related to "vanishing elasticity," and are influenced by similar analyses of Ginzburg-Landau models found in [3] and elsewhere. All three obtain (away from a singular set) limiting uniaxial minimizers of the form (1.3), with constant $S$ determined so as to provide a minimum of $f_{\mathrm{b}}$, and with the director field $\boldsymbol{n}$ corresponding to the minimizer of an appropriate Oseen-Frank model. We show that these limits are properly interpreted not as limits of vanishing elasticity but as limits in which intrinsic length scales (associated with defect core sizes and such) become vanishingly small compared to geometric length scales (associated with the size of the problem domain $\Omega$ ).

The limits analyzed in $[4,13]$ pertain to the behavior of Landau-de Gennes models at low temperatures, where the changing landscape of the bulk freeenergy $f_{\mathrm{b}}$ penalizes biaxial order less and at the same time shrinks defect core sizes. This "low temperature" limit as well corresponds to a "zero core size" limit (with additional features), and the close relationship between these two limits is demonstrated below. Different length scales are known to be associated with defect core sizes in these two different regimes, and it is also shown below how these can be identified via balances in appropriate scalings of the EulerLagrange equations associated with (1.1).

\section{Scaling analysis}

Values of the material parameters in (1.1) are usually found quoted in SI units. For a somewhat typical material, they are roughly in the following ranges:

$$
L \approx 10^{-11} \mathrm{~J} / \mathrm{m}, \quad A, B, C \approx 10^{5} \mathrm{~J} / \mathrm{m}^{3} .
$$

See for example [22, Table 1, p. 168]. We observe that the numerical value of $L$ is 16 orders of magnitude smaller than the values of $A, B$, and $C$ when expressed in these units. If, however, lengths are expressed in units of nanometers (instead of meters), these values become

$$
L \approx 10^{-20} \mathrm{~J} / \mathrm{nm}, \quad A, B, C \approx 10^{-22} \mathrm{~J} / \mathrm{nm}^{3} .
$$

Now the value of $L$ is two orders of magnitude larger than $A, B$, and $C$. The point is that the elastic constants and the bulk constants have different physical dimensions (energy per unit length versus energy per unit volume) and can't be compared. To determine what is "big" versus what is "small," one must non-dimensionalize. An additional point to be made is that as $L \rightarrow 0$, with the model left in dimensional form (as in $[2,16]$ ), one quickly gets beyond the measured values of $L$ for real liquid crystal materials.

\subsection{Large-body limit}

There are numerous ways to non-dimensionalize (1.1), and these depend in general on the particular problem at hand. In essence, in order to form an appropriate dimensionless coupling coefficient between the elastic terms and the bulk terms in the free-energy density, one requires a characteristic elastic 
constant, a characteristic bulk constant, and a characteristic length scale from the geometry of the problem domain. For the purpose of understanding the "vanishing elasticity" limit, a simple rescaling can be done as follows. Let $R$ denote a characteristic geometric length scale. For example, this could be the radius or diameter of a liquid crystal droplet or capillary, or the cell gap of a liquid crystal thin film. Rescale lengths by $R$,

$$
\bar{x}_{i}=x_{i} / R, \quad R=\operatorname{diam}(\Omega),
$$

so that $\nabla=\frac{1}{R} \bar{\nabla}, \mathrm{d} V=R^{3} \mathrm{~d} \bar{V}, \operatorname{diam}(\bar{\Omega})=1$. Let $A_{\mathrm{NI}}$ denote the value of the $A$ parameter at $T=T_{\mathrm{NI}}$, as in (1.5), and take this as our characteristic bulk parameter-other natural candidates for this would be $A_{\mathrm{SH}}$ or $B^{2} / C$, or simply $B$ or $C$. The tensor order parameter $\mathbf{Q}$ is dimensionless by definition, however it is convenient to rescale it as well, as this allows one to eliminate another parameter from the model. Thus we take

$$
\mathbf{Q}=\alpha \overline{\mathbf{Q}}, \quad \alpha:=\frac{1}{\sqrt{27}} \frac{B}{C} .
$$

Other multiples of $B / C$ would work equally well. After simplifying, we obtain

$$
\overline{\mathcal{F}}[\overline{\mathbf{Q}}]=\int_{\bar{\Omega}}\left[\frac{1}{2} \bar{\xi}_{\mathrm{NI}}^{2}|\bar{\nabla} \overline{\mathbf{Q}}|^{2}+\frac{\theta}{2} \operatorname{tr}\left(\overline{\mathbf{Q}}^{2}\right)-\sqrt{3} \operatorname{tr}\left(\overline{\mathbf{Q}}^{3}\right)+\frac{1}{4} \operatorname{tr}\left(\overline{\mathbf{Q}}^{2}\right)^{2}\right] \mathrm{d} \bar{V},
$$

where

$$
\overline{\mathcal{F}}=\frac{\mathcal{F}}{\alpha^{2} A_{\mathrm{NI}} R^{3}}, \bar{\xi}_{\mathrm{NI}}=\frac{\xi_{\mathrm{NI}}}{R}, \xi_{\mathrm{NI}}:=\sqrt{\frac{L}{A_{\mathrm{NI}}}}, \theta:=\frac{A}{A_{\mathrm{NI}}}=\frac{T-T_{\mathrm{SC}}}{T_{\mathrm{NI}}-T_{\mathrm{SC}}} .
$$

The parameter $\xi_{\mathrm{NI}}$ can be interpreted as the "nematic correlation length" at the nematic-isotropic transition temperature $T=T_{\mathrm{NI}}$ and $\theta$ as a reduced temperature, with the corresponding values

$$
T=T_{\mathrm{SC}}, T_{\mathrm{NI}}, T_{\mathrm{SH}} \quad \leftrightarrow \quad \theta=0,1,9 / 8 .
$$

Here and in (2.3), we have used the relations for $A_{\mathrm{NI}}$ and $A_{\mathrm{SH}}$ in (1.5). In a typical liquid-crystal system (for example a thin film of cell gap $R$ containing a low-molecular-weight liquid crystal material), we could have

$$
\xi_{\mathrm{NI}} \approx 10 \mathrm{~nm}, R \approx 10 \mu \mathrm{m} \Rightarrow \bar{\xi}_{\mathrm{NI}} \approx 10^{-3} .
$$

The approximate value for $\xi_{\mathrm{NI}}$ above can be seen to follow roughly from (2.1):

$$
\xi_{\mathrm{NI}}^{2}=\frac{L}{A_{\mathrm{NI}}} \approx \frac{10^{-11} \mathrm{~J} / \mathrm{m}}{10^{5} \mathrm{~J} / \mathrm{m}^{3}}=10^{-16} \mathrm{~m}^{2} .
$$

Such small values of the dimensionless coupling coefficient $\left(\bar{\xi}_{\mathrm{NI}}^{2} \approx 10^{-6}\right)$ would place a large weight on the terms from the bulk ordering potential, strongly encouraging uniaxial order at the bulk-minimizing value of the scalar order parameter. 
The reduced temperature $\theta$ corresponds to the temperature above or below $T_{\mathrm{SC}}$ measured in units of $\left(T_{\mathrm{NI}}-T_{\mathrm{SC}}\right)$, which is approximately the width of the coexistence temperature region (where both the isotropic and the ordered phases are locally stable). For a fairly pure sample of a low-molecular-weight liquid crystal, the coexistence region would typically be one or two degrees Celsius wide, and so one can think of $\theta$ as roughly the temperature above/below $T_{\mathrm{SC}}$ in degrees Celsius. The critical temperatures of liquid crystal materials vary quite a lot. The values of $T_{\mathrm{NI}}$ for three commonly studied liquid crystals are given in $\left[27\right.$, Table D.3, p. 330] as $T_{\mathrm{NI}}=35.4^{\circ} \mathrm{C}(5 \mathrm{CB}), 45.1^{\circ} \mathrm{C}(\mathrm{MBBA})$, $135.5^{\circ} \mathrm{C}$ (PAA). Reports for other experiments on $\mathrm{MBBA}$ give $T_{\mathrm{SC}}=45^{\circ} \mathrm{C}$ and $T_{\mathrm{NI}}=46^{\circ} \mathrm{C}$-see [17, Table I, p. 6695] (note however that the values for $a_{0}$, $B$, and $C$ in this table are in error, transcribed incorrectly from [22, Table 1 , p. 168]). Thus at room temperature (taken as $21^{\circ} \mathrm{C}$ ), we would have $\theta=-24$ for MBBA-while PAA is not even in a liquid crystal phase at room temperature.

The nematic correlation length has a statistical-physics interpretation, however in our continuum model, it simply emerges as a singular-perturbation parameter in the Euler-Lagrange equations associated with (1.1):

$$
-L \Delta \mathbf{Q}+\frac{\partial f_{\mathrm{b}}}{\partial \mathbf{Q}}=\mathbf{0}
$$

Here $\ulcorner$ denotes the symmetric traceless part

$$
\overline{\frac{\partial f_{\mathrm{b}}}{\partial \mathbf{Q}}}=A \mathbf{Q}-B\left[\mathbf{Q}^{2}-\frac{1}{3} \operatorname{tr}\left(\mathbf{Q}^{2}\right) \mathbf{I}\right]+C \operatorname{tr}\left(\mathbf{Q}^{2}\right) \mathbf{Q},
$$

which is associated with the constraints on $\mathbf{Q}$. At $T=T_{\mathrm{NI}}$, we have

$$
-\xi_{\mathrm{NI}}^{2} \Delta \mathbf{Q}+\frac{1}{A_{\mathrm{NI}}} \frac{\overline{\partial f_{\mathrm{b}}}}{\partial \mathbf{Q}}=\mathbf{0}
$$

When $\mathbf{Q}$ is close to a stationary point of $f_{\mathrm{b}}$ (such as $\mathbf{Q}=\mathbf{0}$ or the bottom of a well), then the term $\left(1 / A_{\mathrm{NI}}\right) \overparen{\partial f_{\mathrm{b}} / \partial \mathbf{Q}}$ will be close to zero; otherwise, it will be $O(1)$. The necessary balance between the terms in the equation above indicates how $\xi_{\mathrm{NI}}$ determines the "core size" of a defect (via a scaling of $X_{i}=x_{i} / \xi_{\mathrm{NI}}$ for the "inner solution").

We have defined $\xi_{\mathrm{NI}}$ by linearization around the isotropic state $\mathbf{Q}=\mathbf{0}$, which is always a critical point of $f_{\mathrm{b}}$ and the solution one would see at the center of an isotropic core. Note however that isotropic defect cores are only stable at the high end of the nematic temperature range (see $[10,24])$. The core structure of a defect is generally more complicated than isotropic disordering, and this is discussed more in what follows. In some settings, it is more natural to define the correlation length by linearizing around the nontrivial uniaxial $\mathbf{Q}$ tensor that gives the global minimum of $f_{\mathrm{b}}$ for $T<T_{\mathrm{NI}}$ (see for example [23]). Correlation lengths and core sizes depend on temperature, and this should be taken into account in non-dimensionalizations if one intends to explore behavior deep in the nematic phase-we do this below. In some situations, a more natural intrinsic length scale is given by a "biaxial coherence length" (see [14,21])-we 
consider this as well in what follows. The scaling we have adopted above has been chosen for simplicity. It is by no means original. Similar scalings are employed by all practitioners who work with Landau-de Gennes models. In addition to references already cited, see for example $[6,8,11,17,26,30]$.

In our rescaled free energy (2.3), all quantities are dimensionless; the size of $\bar{\Omega}$ is $O(1)$; and at the high end of the nematic temperature range, $\overline{\mathcal{F}}, \overline{\mathbf{Q}}$, and $\theta$ are $O(1)$ as well. We can see under what circumstances the coupling coefficient between the elastic and bulk terms is small:

$$
0<\bar{\xi}_{\mathrm{NI}} \ll 1 \Leftrightarrow 0<\xi_{\mathrm{NI}} \ll R
$$

that is, when the nematic correlation length (isotropic core size) is small compared to a length scale associated with the problem geometry (size of $\Omega$ ). The dimensionless spatial gradient $\bar{\nabla} \overline{\mathbf{Q}}$ will be $O(1)$ away from defects and $O\left(1 / \bar{\xi}_{\mathrm{NI}}\right)$ in the vicinity of defects. The limit $\bar{\xi}_{\mathrm{NI}} \rightarrow 0$ corresponds to the core size of defects becoming vanishingly small compared to the size of the problem geometry, with finite-size defects becoming point or line singularities in the limit. This can be thought of as a "zero-core-size limit" or a "large-body limit."

For the more general functional (1.7) (without the artificially introduced $\varepsilon$ ), a similar rescaling would give

$$
\begin{aligned}
\overline{\mathcal{F}}[\overline{\mathbf{Q}}]=\int_{\bar{\Omega}}\left[\frac{1}{2} \bar{\xi}_{\mathrm{NI}}^{2}\left(\bar{Q}_{i j, \bar{k}} \bar{Q}_{i j, \bar{k}}+\bar{L}_{2} \bar{Q}_{i j, \overline{\bar{Q}}} \bar{Q}_{i k, \bar{k}}+\bar{L}_{3} \bar{Q}_{i j, \bar{k}} \bar{Q}_{i k, \bar{j}}\right)\right. \\
\left.+\frac{\theta}{2} \operatorname{tr}\left(\overline{\mathbf{Q}}^{2}\right)-\sqrt{3} \operatorname{tr}\left(\overline{\mathbf{Q}}^{3}\right)+\frac{1}{4} \operatorname{tr}\left(\overline{\mathbf{Q}}^{2}\right)^{2}\right] \mathrm{d} \bar{V}
\end{aligned}
$$

where

$$
\bar{\xi}_{\mathrm{NI}}=\frac{\xi_{\mathrm{NI}}}{R}, \quad \xi_{\mathrm{NI}}:=\sqrt{\frac{L_{1}}{A_{\mathrm{NI}}}}, \quad \bar{L}_{2}:=\frac{L_{2}}{L_{1}}, \quad \bar{L}_{3}:=\frac{L_{3}}{L_{1}} .
$$

Here $\bar{L}_{2}$ and $\bar{L}_{3}$ are dimensionless and $O(1)$. The definition of the dimensionless parameter $\varepsilon$ in (1.8) is not given in [12]. One can assume that it has been constructed in a way that is analogous to what has been done here for $\bar{\xi}_{\mathrm{NI}}$ in (2.3) and above.

\subsection{Low-temperature limit}

In the "low temperature" regime, deep in the nematic phase, several effects are manifested by the Landau-de Gennes models and their free-energy-minimizing solutions. These include the degree of orientational order increasing, the potential wells in $f_{\mathrm{b}}$ becoming deeper with the barriers between the wells smaller, and correlation lengths and defect core sizes becoming smaller as well. These various features must be taken into account in a good scaling of the free-energy functional for this range. The combination of these features serves to penalize biaxiality less, encouraging localized biaxiality more as a way for equilibrium tensor fields to avoid the large free energy costs of isotropic cores in defects, and this is the motivation for papers such as $[4,13]$ to analyze this limit rigorously.

Recall that the only temperature dependence of the parameters in (1.1) is through the $A$ parameter, assumed to have the form $A=a_{0}\left(T-T_{\mathrm{SC}}\right), a_{0}$ a 
positive constant, given in (1.2). Thus $A$ becomes more and more negative, deeper and deeper into the nematic phase. The behavior of the scalar order parameter $S$ and bulk potential $f_{\mathrm{b}}$ in this range can be determined as follows. Under the uniaxial assumption (1.3), one obtains

$$
\mathbf{Q}=S\left(\boldsymbol{n} \otimes \boldsymbol{n}-\frac{1}{3} \mathbf{I}\right) \Rightarrow f_{\mathrm{b}}(\mathbf{Q})=\frac{A}{3} S^{2}-\frac{2 B}{27} S^{3}+\frac{C}{9} S^{4}=: f(S),
$$

for which the nontrivial critical points are given by

$$
S_{ \pm}=\frac{B \pm \sqrt{B^{2}-24 A C}}{4 C} \approx \pm \sqrt{\frac{-3 A}{2 C}}, \quad \frac{A}{C} \ll-1,
$$

with associated values $f\left(S_{ \pm}\right) \approx-A^{2} /(4 C), A / C \ll-1$. The dimensionless ratio $A / C$ is proportional to the reduced temperature $\theta$ used previously:

$$
\frac{A}{C}=\frac{A_{\mathrm{NI}}}{C} \frac{A}{A_{\mathrm{NI}}}=\frac{A_{\mathrm{NI}}}{C} \theta, \quad \theta=\frac{A}{A_{\mathrm{NI}}} \text { as in }(2.3 \mathrm{~b}) .
$$

Here the ratio $A_{\mathrm{NI}} / C$ is dimensionless and $O(1)$, and we see that

$$
\left|S_{ \pm}\right|=O(\sqrt{-\theta}), \quad-\frac{1}{C} f\left(S_{ \pm}\right)=O\left(\theta^{2}\right), \quad \text { as } \theta \rightarrow-\infty .
$$

We will rescale our single-elastic-constant Landau-de Gennes model (1.1) for the low-temperature regime using this information and staying as close as possible to the parameter definitions and notations used in the preceding subsection.

We scale lengths as before in (2.2), using some length scale $R$ appropriate to the geometry of the problem domain: $\bar{x}_{i}=x_{i} / R, \bar{\Omega}=\Omega / R$. We scale $\mathbf{Q}$ and $\mathcal{F}$ by their low-temperature asymptotic values, given in $(2.5)$ :

$$
\mathbf{Q}=\sqrt{\frac{-A}{C}} \widetilde{\mathbf{Q}}, \quad \mathcal{F}=\frac{A^{2}}{C} R^{3} \widetilde{\mathcal{F}} .
$$

Introducing these scalings into (1.1) and simplifying, we obtain

$$
\widetilde{\mathcal{F}}[\widetilde{\mathbf{Q}}]=\int_{\bar{\Omega}}\left[\frac{1}{2} \varepsilon^{2} \bar{\xi}_{\mathrm{NI}}^{2}|\bar{\nabla} \widetilde{\mathbf{Q}}|^{2}-\frac{1}{2} \operatorname{tr}\left(\widetilde{\mathbf{Q}}^{2}\right)-\sqrt{3} \varepsilon \operatorname{tr}\left(\widetilde{\mathbf{Q}}^{3}\right)+\frac{1}{4} \operatorname{tr}\left(\widetilde{\mathbf{Q}}^{2}\right)^{2}\right] \mathrm{d} \bar{V},
$$

where

$$
\varepsilon:=\frac{1}{\sqrt{-\theta}}, \quad \theta=\frac{A}{A_{\mathrm{NI}}}=\frac{T-T_{\mathrm{SC}}}{T_{\mathrm{NI}}-T_{\mathrm{SC}}}, \quad \bar{\xi}_{\mathrm{NI}}=\frac{\xi_{\mathrm{NI}}}{R}, \quad \xi_{\mathrm{NI}}=\sqrt{\frac{L}{A_{\mathrm{NI}}}} .
$$

Here the definitions of $\theta, \xi_{\mathrm{NI}}$, and $\bar{\xi}_{\mathrm{NI}}$ are exactly as before, and we have adopted the small parameter $\varepsilon$ as a control parameter:

$$
\theta \ll-1 \leftrightarrow 0<\varepsilon \ll 1 .
$$

We have deliberately written the temperature-dependent nematic correlation length, $\xi_{\mathrm{n}}$, in terms of $\varepsilon$ and the nematic correlation length at $T=T_{\mathrm{NI}}\left(\xi_{\mathrm{NI}}\right.$, defined in $(2.3 \mathrm{~b}))$ :

$$
\xi_{\mathrm{n}}:=\sqrt{\frac{L}{-A}}=\sqrt{\frac{A_{\mathrm{NI}}}{-A}} \sqrt{\frac{L}{A_{\mathrm{NI}}}}=\frac{1}{\sqrt{-\theta}} \xi_{\mathrm{NI}}=\varepsilon \xi_{\mathrm{NI}} .
$$


The relevance of $\xi_{\mathrm{n}}$ can already be gleaned from the coupling between the terms $\bar{\xi}_{\mathrm{NI}}^{2}|\bar{\nabla} \overline{\mathbf{Q}}|^{2}$ and $\theta \operatorname{tr}(\overline{\mathbf{Q}})^{2}$ in $(2.3 \mathrm{a})$. We can see above how the correlation length scales with temperature, shrinking like $1 / \sqrt{-\theta}$ as $\theta$ becomes more and more negative. In (2.7a), we have again used the relation $A_{\mathrm{NI}}=B^{2} / 27 C$ from (1.5) to simplify the coefficient of the $\operatorname{tr}\left(\mathbf{Q}^{3}\right)$ term.

The relationship between the "large body" scaling, in (2.3) and (2.4), and the "low temperature" scaling in (2.7) is given by

$$
\overline{\mathbf{Q}}=\sqrt{-\theta} \widetilde{\mathbf{Q}}, \quad \overline{\mathcal{F}}=\theta^{2} \widetilde{\mathcal{F}},
$$

which can be verified directly from the definitions of $\overline{\mathbf{Q}}, \overline{\mathcal{F}}, \widetilde{\mathbf{Q}}, \widetilde{\mathcal{F}}$, and $\theta$, with the help of $A_{\mathrm{NI}}=B^{2} / 27 C$ from (1.5). One can, in fact, derive (2.7) directly from (2.3) by introducing the relations (2.9) into (2.3) and simplifying. We note that (2.3) and (2.4) are not good scalings for exploring the low-temperature limit $(\theta \rightarrow-\infty)$, because they do not take into account the behavior of $|\overline{\mathbf{Q}}|$ in that range:

$$
\operatorname{tr}\left(\overline{\mathbf{Q}}^{2}\right)=O(|\theta|), \quad \operatorname{tr}\left(\overline{\mathbf{Q}}^{3}\right)=O\left(|\theta|^{3 / 2}\right), \quad \operatorname{tr}\left(\overline{\mathbf{Q}}^{2}\right)^{2}=O\left(|\theta|^{2}\right), \quad \text { as } \theta \rightarrow-\infty .
$$

In our free-energy functional rescaled for the low-temperature regime, (2.7), all quantities are again dimensionless; the size of $\bar{\Omega}$ is again $O(1)$; and for $\theta \leq-1(0<\varepsilon \leq 1), \widetilde{\mathcal{F}}$ and $\widetilde{\mathbf{Q}}$ are $O(1)$ as well. The non-dimensionalized model shares many features with the functional (2.3), which is scaled for the large-body regime. Both are left with the same two dimensionless parameters, $\bar{\xi}_{\mathrm{NI}}$ (the ratio of the nematic correlation length at $T=T_{\mathrm{NI}}$ to the geometric length scale $R$ ) and the reduced temperature $\theta$ (which enters (2.7) through $\varepsilon=1 / \sqrt{-\theta})$. In $(2.3), \theta$ can be positive, negative, or zero, which allows for using that scaling up to and including the co-existence temperature range $(0 \leq \theta \leq 9 / 8)$, as in [30]; while in (2.7), $\theta<0$ is assumed-the two scaled models are identical, in fact, when $\theta=-1$ (for any $\bar{\xi}_{\mathrm{NI}}$ ). The models share the feature of a dimensionless coupling coefficient between the elastic and the bulk terms $\left(\bar{\xi}_{\mathrm{NI}}^{2}\right.$ in $(2.3), \varepsilon^{2} \bar{\xi}_{\mathrm{NI}}^{2}$ in $\left.(2.7)\right)$, and these coupling coefficients are expected to be quite small in the respective regimes of interest. Both the large-body limit explored in $[2,12,16]\left(\bar{\xi}_{\mathrm{NI}} \rightarrow 0\right)$ and the low-temperature limit in $[4,13](\varepsilon \rightarrow 0)$ correspond to circumstances in which the (temperature dependent) core size of defects becomes vanishingly small compared to the size of the domain-a "zero-relative-core-size limit."

The presence of the factor $\varepsilon$ in the coefficient of the $\operatorname{tr}\left(\widetilde{\mathbf{Q}}^{3}\right)$ term in $(2.7)$,

$$
\tilde{f}_{\mathrm{b}, \varepsilon}(\widetilde{\mathbf{Q}})=-\frac{1}{2} \operatorname{tr}\left(\widetilde{\mathbf{Q}}^{2}\right)-\sqrt{3} \varepsilon \operatorname{tr}\left(\widetilde{\mathbf{Q}}^{3}\right)+\frac{1}{4} \operatorname{tr}\left(\widetilde{\mathbf{Q}}^{2}\right)^{2}
$$

drives that term to zero in the low-temperature limit and leaves the rescaled bulk ordering potential in the form

$$
\tilde{f}_{\mathrm{b}, 0}(\widetilde{\mathbf{Q}})=-\frac{1}{2} \operatorname{tr}\left(\widetilde{\mathbf{Q}}^{2}\right)+\frac{1}{4} \operatorname{tr}\left(\widetilde{\mathbf{Q}}^{2}\right)^{2} .
$$

Thus $\tilde{f}_{\mathrm{b}, 0}$ is expressed only in terms of even powers, $\operatorname{tr}\left(\widetilde{\mathbf{Q}}^{2}\right)$ and $\operatorname{tr}\left(\widetilde{\mathbf{Q}}^{2}\right)^{2}$, and more closely resembles the types of Landau expansions found in the GinzburgLandau theory of superconductivity. The potential $\tilde{f}_{\mathrm{b}, 0}$ has a local maximum 
value of $\tilde{f}_{\mathrm{b}, 0}=0$ at the isotropic state $\widetilde{\mathbf{Q}}=\mathbf{0}$ and absolute minima given by

$$
\min \tilde{f}_{\mathrm{b}, 0}=-\frac{1}{4}, \text { when } \operatorname{tr}\left(\widetilde{\mathbf{Q}}^{2}\right)=1
$$

The distinguishing feature here is that the set of bulk-minimizing tensor order parameters $\left(\widetilde{\mathbf{Q}}\right.$ satisfying $\operatorname{tr}\left(\widetilde{\mathbf{Q}}^{2}\right)=1$ ) includes a continuum of biaxial states (in addition to uniaxial states of the form (1.3)). Thus the usual penalty from $f_{\mathrm{b}}$ for biaxiality vanishes in this limit. This behavior has been known for some time (see [15]). It is the basis of the so-called "Lyuksyutov constraint" $\left(\operatorname{tr}\left(\mathbf{Q}^{2}\right)=\right.$ const $)$, which has been used on occasion to obtain approximations from Landau-de Gennes models deep in the nematic phase-see for example $[14,15,21]$. The justification of the Lyuksyutov constraint is well illustrated by (2.10), and this behavior also underlies the analyses in $[4,13]$.

The main thrust of [4] is to prove that at sufficiently low temperatures, tensor fields that minimize the Landau-de Gennes free energy (1.1a) do not have isotropic cores (even if boundary conditions dictate that a defect of some kind must be present). Such tensor fields avoid the large free-energy cost of such cores by going through localized biaxial transitions instead. The paper [13] provides some generalizations of [4] and focuses some consideration on the "vanishing elasticity limit" aspect. Both papers use some rescaling but leave the free-energy functionals in partly dimensional form: the main integrals ((10) in [4], (14) in [13]) have the physical dimensions of a volume and the coupling coefficients are lengths squared. One can establish some connections with the low-temperature scaling we have adopted here in (2.7). Both [4] and [13] scale $\mathrm{Q}$ by the temperature-dependent scalar order parameter $\sqrt{2 / 3} S_{+}$, whereas here we have scaled $\mathbf{Q}$ by the asymptotic value of this,

$$
\sqrt{\frac{2}{3}} S_{+} \approx \sqrt{\frac{-A}{C}}, \text { for } \frac{A}{C} \ll-1,
$$

from (2.5a) and (2.6). The reduced temperatures, denoted by $t$ in both papers (but defined slightly differently in each), are related to $\theta$ in (2.3b) here via

$$
t=-\frac{1}{27} \theta \text { in [4], } t=-\theta \text { in [13] }
$$

and the coupling coefficients are related to $\xi_{\mathrm{NI}}$ in $(2.3 \mathrm{~b})$ here by

$$
\widetilde{L}=\frac{1}{9} \xi_{\mathrm{NI}}^{2} \text { in }[4], \quad \bar{L}=\frac{1}{2} \xi_{\mathrm{NI}}^{2} \text { in }[13]
$$

In both papers, the rescaled integral functionals are parameterized by the large parameter $t$, rather than the small parameter $\varepsilon$ we have used here. If one were to scale the integral (10) in [4] or (14) in [13] by $1 / t$ and non-dimensionalize the lengths, then one would arrive at something close to (2.7) here. The scaling that we have adopted here also enables us to make contact with some aspects of biaxiality in the low-temperature regime, which we discuss next. 


\subsection{Biaxiality in the low-temperature regime}

The biaxial nature of defects in typical temperature ranges (as modeled in the Landau-de Gennes framework) was demonstrated in [25], based upon earlier predictions. The size of such "biaxial cores" is known to be determined by the (temperature-dependent) "biaxial coherence length," defined in [14,21] as $\xi_{\mathrm{b}}:=$ $\sqrt{L /\left(B S_{+}\right)}$. In the low-temperature regime, this can be related to parameters we have used elsewhere in this note (using formulas in (1.5) and (2.7b)):

$$
S_{+} \approx \sqrt{\frac{-3 A}{2 C}}, \quad \frac{A}{C} \ll-1 \Rightarrow \xi_{\mathrm{b}}=\sqrt{\frac{L}{B S_{+}}} \approx \frac{1}{3} 2^{1 / 4} \varepsilon^{1 / 2} \xi_{\mathrm{NI}}, \quad 0<\varepsilon \ll 1 .
$$

Observe the difference in the temperature dependence of $\xi_{\mathrm{b}}$ versus that of the nematic correlation length $\xi_{\mathrm{n}}$ :

$$
\xi_{\mathrm{b}}=\frac{1}{3} 2^{1 / 4} \varepsilon^{1 / 2} \xi_{\mathrm{NI}}(1+O(\varepsilon)), \text { as } \varepsilon \rightarrow 0+\quad \text { vs } \quad \xi_{\mathrm{n}}=\varepsilon \xi_{\mathrm{NI}}, \quad \text { from }(2.8) .
$$

At high temperatures, however, these lengths are essentially equivalent:

$$
A_{\mathrm{NI}}=\frac{B^{2}}{27 C}, S_{\mathrm{NI}}=\frac{B}{3 C} \Rightarrow \xi_{\mathrm{n}}=\sqrt{\frac{L}{A_{\mathrm{NI}}}}=3 \sqrt{\frac{L}{B S_{\mathrm{NI}}}}=3 \xi_{\mathrm{b}}, \text { at } T=T_{\mathrm{NI}} .
$$

We will see below that the biaxial coherence length can be identified from a balance in the equilibrium Euler-Lagrange equations for $\mathbf{Q}$ for the "inner solution" in a biaxial defect core.

There is a subtle mechanism by which biaxiality can enable defects that cost less free energy than isotropic cores. This has been explored in several papers, including $[6,10,14,17,20,21,25,26]$. It can be described loosely as follows. Recall that the values of the bulk free-energy density $f_{\mathrm{b}}$ depend only on the eigenvalues of $\mathbf{Q}$. If one studies the surface of $f_{\mathrm{b}}$ as a function of the two independent eigenvalues of $\mathbf{Q}$ (parameterized as in (1.4), with eigenframe $\boldsymbol{e}_{1}$, $\left.\boldsymbol{e}_{2}, \boldsymbol{e}_{3}\right)$, then one finds three wells, at positively ordered uniaxial states of the form (1.3) with $S=S_{+}>0\left(S_{+}\right.$as given in (2.5a)), each well corresponding to a different director $\boldsymbol{n}=\boldsymbol{e}_{1}, \boldsymbol{e}_{2}, \boldsymbol{e}_{3}$ (the solid circles in Figure 1). These three global minima are separated from each other by saddle points, which also correspond to uniaxial states but which are negatively ordered, with $S=$ $S_{-}<0$ (again from (2.5a), the crosses in Figure 1). An excursion by the eigenvalues of $\mathbf{Q}$ from one well to another thus enables a reorientation of $\boldsymbol{n}$ without rotating the eigenframe of $\mathbf{Q}$. This mechanism is sometimes referred to as "eigenvalue exchange," and it is the mechanism at work in a "hybrid cell" at extremely narrow cell gap [20]. A hybrid cell is a thin film of liquid crystal sandwiched between substrates that are treated to coerce alignment of the director parallel to one substrate and perpendicular to the other. At a sufficiently small thickness of a hybrid cell, eigenvalue exchange has a lower free energy than the competing $\pm \pi / 2$ rotation of the director. In geometries that encourage a point defect or a disclination line (or such), instead of an isotropic defect core, there typically would form a small structure (such as a ring or torus) across which such an eigenvalue-exchange transition would 
occur $[6,10,14,17,21,26]$. With the exception of the wells and the saddle points, all other points along the path of such an eigenvalue excursion would be biaxial.

A significant component of the cost of such a "biaxial escape" is climbing the potential barrier to cross the "mountain pass" between two wells. In the lowtemperature regime, however, the magnitude of this potential barrier becomes smaller, which can be seen as follows. In the low-temperature scaling (2.10) (with $\widetilde{\mathbf{Q}}$ as defined in (2.6)), the critical scalar order parameters (rescaled analogues of $(2.5 \mathrm{a})$ for $\left.\tilde{f}_{\mathrm{b}, \varepsilon}(\widetilde{\mathbf{Q}})\right)$ are given by

$$
\widetilde{S}_{ \pm}=\frac{3 \sqrt{3} \varepsilon \pm \sqrt{27 \varepsilon^{2}+24}}{4}= \pm \sqrt{\frac{3}{2}}+\frac{3 \sqrt{3}}{4} \varepsilon+O\left(\varepsilon^{2}\right) .
$$

Denoting $\widetilde{\mathbf{Q}}_{ \pm}=\widetilde{S}_{ \pm}(\boldsymbol{n} \otimes \boldsymbol{n}-\mathbf{I} / 3)$, we obtain

$$
\tilde{f}_{\mathrm{b}, \varepsilon}\left(\widetilde{\mathbf{Q}}_{ \pm}\right)=-\frac{1}{4} \mp \frac{1}{\sqrt{2}} \varepsilon+O\left(\varepsilon^{2}\right) \quad \text { and } \quad \operatorname{tr}\left(\widetilde{\mathbf{Q}}_{ \pm}^{2}\right)=1 \pm \frac{3}{\sqrt{2}} \varepsilon+O\left(\varepsilon^{2}\right)
$$

With the eigenvalues of $\widetilde{\mathbf{Q}}$ taking the path of least resistance along such an excursion between wells, we would then have

$$
\tilde{f}_{\mathrm{b}, \varepsilon}\left(\widetilde{\mathbf{Q}}_{+}\right) \leq \tilde{f}_{\mathrm{b}, \varepsilon}(\widetilde{\mathbf{Q}}) \leq \tilde{f}_{\mathrm{b}, \varepsilon}\left(\widetilde{\mathbf{Q}}_{-}\right), \tilde{f}_{\mathrm{b}, \varepsilon}\left(\widetilde{\mathbf{Q}}_{-}\right)-\tilde{f}_{\mathrm{b}, \varepsilon}\left(\widetilde{\mathbf{Q}}_{+}\right)=\sqrt{2} \varepsilon+O\left(\varepsilon^{2}\right)
$$

and

$$
\operatorname{tr}\left(\widetilde{\mathbf{Q}}_{-}^{2}\right) \leq \operatorname{tr}\left(\widetilde{\mathbf{Q}}^{2}\right) \leq \operatorname{tr}\left(\widetilde{\mathbf{Q}}_{+}^{2}\right) \Rightarrow\left|\operatorname{tr}\left(\widetilde{\mathbf{Q}}^{2}\right)-1\right| \leq \frac{3}{\sqrt{2}} \varepsilon+O\left(\varepsilon^{2}\right) .
$$

The $O(\varepsilon)$ potential barrier seen in (2.11a) is much more favorable than the cost of isotropic melting: $\tilde{f}_{\mathrm{b}, \varepsilon}(\mathbf{0})-\tilde{f}_{\mathrm{b}, \varepsilon}\left(\widetilde{\mathbf{Q}}_{+}\right)=1 / 4+O(\varepsilon)$. A smooth $O(\varepsilon)$ change in $\tilde{f}_{\mathrm{b}, \varepsilon}$ (consistent with (2.11a)) over the course of an $O(1)$ change in $\widetilde{\mathbf{Q}}$ implies $\partial \tilde{f}_{\mathrm{b}, \varepsilon} / \partial \widetilde{\mathbf{Q}}=O(\varepsilon)$ along such a path, and this can be seen from the Euler-Lagrange equations in this scaling:

$$
-\varepsilon^{2} \bar{\xi}_{\mathrm{NI}}^{2} \bar{\Delta} \widetilde{\mathbf{Q}}+\left[\operatorname{tr}\left(\widetilde{\mathbf{Q}}^{2}\right)-1\right] \widetilde{\mathbf{Q}}-3 \sqrt{3} \varepsilon\left[\widetilde{\mathbf{Q}}^{2}-\frac{1}{2} \operatorname{tr}\left(\widetilde{\mathbf{Q}}^{2}\right) \mathbf{I}\right]=\mathbf{0} .
$$

The middle and last terms above are both seen to be $O(\varepsilon)$ (with the help of $(2.11 b)$ ), and the necessary balance that must exist in the "interior layer" between these terms and the leading term above gives a length scale in the biaxial core of $\varepsilon^{1 / 2} \bar{\xi}_{\mathrm{NI}}$ (which is the low-temperature asymptotic value of $\bar{\xi}_{\mathrm{b}}$ ). This dependence on reduced temperature $\left(\varepsilon^{1 / 2}=(-\theta)^{-1 / 4}\right)$ is consistent with results in $[4,13]$, where some of these same quantities of interest are considered in a more rigorous way. While biaxial cores are somewhat larger than isotropic cores and shrink more slowly than isotropic cores as temperature is reduced, they still become vanishingly small in either the large-body limit or the lowtemperature limit, as do the biaxial rings and tori and such. 


\section{Conclusions}

The Landau-de Gennes model is a mesoscopic model that contains intrinsic length scales of molecular order associated with features such as core sizes of point defects and disclination lines. The Oseen-Frank model, on the other hand, is a macroscopic model and contains no such intrinsic length scales: the defects of equilibrium director fields in Oseen-Frank models are point or line singularities. The scaling analysis in Section 2 shows that the limits explored in $[2,4,12,13,16]$ concern the passage from a mesoscopic model to a macroscopic model as the geometric length scales become large compared to the intrinsic length scales. In such limits, core sizes (isotropic or biaxial) become zero. The situation is analogous to a "London limit" for Ginzburg-Landau models of superconductivity, the simplest example of which is

$$
E_{\varepsilon}[u]=\frac{1}{2} \int_{\Omega}|\nabla u|^{2} \mathrm{~d} A+\frac{1}{4 \varepsilon^{2}} \int_{\Omega}\left(|u|^{2}-1\right)^{2} \mathrm{~d} A, \quad \Omega \subset \mathbb{R}^{2},
$$

as studied in [3]. Here $u$ is a complex-valued order parameter field (with $|u|^{2}$ giving a normalized local density of superconducting electron pairs), and $\varepsilon$ comes from a ratio of length scales. In the limit $\varepsilon \rightarrow 0$, one approaches the "London approximation," in which $|u|=1$ throughout $\Omega$. The limits we have examined here are also related to the "large body" limit of the Landau-Lifshitz free energy in micromagnetics, which can be written

$$
F_{\mathrm{LL}}[\boldsymbol{m}]=\int_{\Omega}\left[\frac{1}{2} C_{\mathrm{ex}}|\nabla \boldsymbol{m}|^{2}+\varphi(\boldsymbol{m})-\mu_{0} M_{\mathrm{s}} \boldsymbol{H}_{\mathrm{e}} \cdot \boldsymbol{m}\right] \mathrm{d} V+\frac{1}{2} \mu_{0} \int_{\mathbb{R}^{3}}\left|\boldsymbol{H}_{\mathrm{S}}\right|^{2} \mathrm{~d} V .
$$

Here $\boldsymbol{m}$ denotes the normalized magnetization field $\left(\boldsymbol{M}=M_{\mathrm{s}} \boldsymbol{m},|\boldsymbol{m}|=1, M_{\mathrm{s}}\right.$ the saturation magnetization constant), $C_{\mathrm{ex}}$ is the exchange constant, $\varphi$ is the anisotropy energy density, $\mu_{0}$ is the vacuum magnetic permeability, $\boldsymbol{H}_{\mathrm{e}}$ is the external applied magnetic field, and $\boldsymbol{H}_{\mathrm{s}}$ is the magnetic stray field generated by $\boldsymbol{M}$. The function $\varphi$ serves to encourage the alignment of $\boldsymbol{m}$ with certain preferred directions. A simple example is $\varphi=K\left(\boldsymbol{m} \cdot \boldsymbol{m}_{0}\right)^{2} / 2$, which encourages $\boldsymbol{m} \perp \boldsymbol{m}_{0}$ (for constant $K>0$ ). See [7]. The physical dimensions of $C_{\mathrm{ex}}$ are energy per unit length, while those of $K$ are energy per unit volume. Thus an intrinsic length scale arises from $\sqrt{C_{\mathrm{ex}} / K}$, much in the same way that $\xi_{\mathrm{NI}}$ and $\xi_{\mathrm{n}}$ arise in $(2.3 \mathrm{~b}),(2.4 \mathrm{~b})$, and $(2.8)$. The common feature here is intrinsic length scales arising from couplings between gradient terms and bulk terms.

The analyses in the papers $[2,4,13,16]$ remain valid after a re-interpretation is done of the problem parameters (into appropriate dimensionless forms)-the functionals in (1.1a) versus (2.3a) and (1.7a) versus (2.4a) involve the same terms in a formal sense, just differently scaled. One should take heed, however, of the pitfalls of analyzing such a physical model in fully (or partially) dimensional form. The numerical values of quantities of different physical dimensions can change their relative sizes when the system of units is changed. It is also the case that familiar Sobolev-type norms, such as

$$
\|\mathbf{Q}\|_{1}^{2}=\int_{\Omega}\left[|\nabla \mathbf{Q}|^{2}+|\mathbf{Q}|^{2}\right] \mathrm{d} V
$$


(which are used in most of the papers in this area), can't even be used in this form unless lengths have been non-dimensionalized; otherwise the first term in the integrand above would have dimensions of the reciprocal of length squared, while the second term would be dimensionless (and the two terms couldn't be combined). We acknowledge that [5] is guilty of this error as well. A remedy for this would be to use weighted Sobolev norms, such as

$$
\|\mathbf{Q}\|_{1}^{2}=\int_{\Omega}\left[w_{1}|\nabla \mathbf{Q}|^{2}+w_{2}|\mathbf{Q}|^{2}\right] \mathrm{d} V
$$

with $w_{1}$ and $w_{2}$ chosen with dimensions to render the combination meaningful. An additional difficulty of attempting to analyze such limits in dimensional form is that as $L \rightarrow 0$ in (1.1a), one quickly gets beyond the measured values of $L$ for real liquid crystal materials.

While the analyses in $[2,4,12,13,16]$ follow earlier work by others on models with some similar features (such as Ginzburg-Landau), technical challenges accompany the analysis of Landau-de Gennes models by virtue of the tensorial nature of the state variable and the multiple terms and parameters and the complexity of the functionals. An effort to address some of the issues we have taken up here was made in a brief appendix in [19]. We note that both of these limits ("large body" and "low temperature") are idealizations from a physical point of view. When the size of the domain containing the liquid crystal becomes too large (compared to intrinsic length scales), thermal fluctuations would wash out any orientational order that other factors might try to induce. Also, at sufficiently low temperatures, all liquid crystal materials would eventually crystallize or transition to a glass or to a non-nematic liquid crystal phase (and both the Landau-de Gennes and Oseen-Frank models would no longer be valid). The study of such limits, however, can give useful insights.

One of the interesting aspects of the analyses done in the papers $[2,4,12$, $13,16]$ is that they provide, to some extent, a justification for using the OseenFrank model to compute equilibrium director fields in the case when line disclinations are present: even though the Oseen-Frank free energy of such solutions is infinite, the equilibrium director fields (found from the Euler-Lagrange equations) are the limiting director fields associated with solutions of a Landaude Gennes model, which solutions have finite free energy for all positive values of the coupling coefficients, though diverging in the limit as the coupling coefficients approach zero. However, the divergence of the Oseen-Frank free-energy functional $F$ evaluated on any director field containing a disclination line prevents it from being used to assess local or global stability of such solutions.

In all but the simplest of settings, one must resort to numerical methods to approximate solutions of Landau-de Gennes or Oseen-Frank models, and this sometimes introduces aspects that are closely related to issues we have highlighted here. An example of this is the use of "penalty methods" for OseenFrank models (or for the related Ericksen-Leslie hydrodynamic equations)see $[1,29]$. This popular approach avoids imposing the constraint $|\boldsymbol{n}|=1$ pointwise (and the necessary introduction of an associated Lagrange-multiplier field) by instead adding a term to the free-energy functional that penalizes departures from $|\boldsymbol{n}|=1$. If the model (1.6) were to be left in dimensional 
form, then the penalized version of it could be written

$$
F_{\xi}[\boldsymbol{n}]=\frac{K}{2} \int_{\Omega}\left[|\nabla \boldsymbol{n}|^{2}+\xi^{-2}\left(|\boldsymbol{n}|^{2}-1\right)^{2}\right] \mathrm{d} V .
$$

The purely numerical "penalty parameter" $\xi$ must have the physical dimensions of a length, and the macroscopic model now has the complexion of a mesoscopic model. The smaller $\xi$ is (compared to the size of the domain, say), the greater the extent to which the constraint is imposed. Equilibria of $F_{\xi}$ that possess defects would have finite "cores" of size $O(\xi)$ (in which the length of $\boldsymbol{n}$ would depart significantly from one). The non-dimensionalized version of the penalized functional above (lengths scaled by $R$, energy by $K R$ ) is

$$
\bar{F}_{\bar{\xi}}[\boldsymbol{n}]=\frac{1}{2} \int_{\bar{\Omega}}\left[|\bar{\nabla} \boldsymbol{n}|^{2}+\bar{\xi}^{-2}\left(|\boldsymbol{n}|^{2}-1\right)^{2}\right] \mathrm{d} \bar{V},
$$

which in two space dimensions is equivalent to the Ginzburg-Landau functional (3.1). All of the Ginzburg-Landau analytical machinery would then apply and give analogous strong convergence results (away from singular sets) for minimizers of $\overline{F_{\bar{\xi}}}$, as the dimensionless penalty parameter $\bar{\xi} \rightarrow 0$. Less appears to be known in the setting of dynamics (liquid crystal director dynamics or full Ericksen-Leslie hydrodynamics). Numerical discretization of such problems would introduce another length scale into the discretized model, the mesh size or grid size (typically denoted by $h$ ), and one would need to take into consideration different regimes of dimensionless ratios of $h, \xi$, and $R$.

Landau-de Gennes and Oseen-Frank models contain a large number of physical parameters, and parameter-dependent effects (changes of state of solutions, bifurcations, structural phase transitions, behaviors in extreme ranges of parameters) are of significant importance in general. Dimensional analysis, scaling, and balances (as used in boundary/interior-layer theory) provide a useful means to understand and interpret some of these behaviors. They have enabled us here to determine the proper interpretation of "vanishing-elasticity limits" and to see one way that length scales for isotropic cores and biaxial cores can be deduced. These simple tools provide a useful complement to approaches coming from the realm of mathematical analysis.

\section{Acknowledgement}

We are grateful to several individuals for feedback on a first version of this note [9], in particular to John Ball, Dmitry Golovaty, Apala Majumdar, André Sonnet, Noel Walkington, and Arghir Zarnescu. We are also grateful to the referees for their careful reading and suggestions.

\section{References}

[1] J.H. Adler, D.B. Emerson, S.P. MacLachlan and T.A. Manteuffel. Constrained optimization for liquid crystal equilibria. SIAM Journal on Scientific Computing, 38(1):B50-B76, 2016. https://doi.org/10.1137/141001846. 
[2] P. Bauman, J. Park and D. Phillips. Analysis of nematic liquid crystals with disclination lines. Archive for Rational Mechanics and Analysis, 205(3):795-826, 2012. https://doi.org/10.1007/s00205-012-0530-7.

[3] F. Bethuel, H. Brezis and F. Hélein. Asymptotics for the minimization of a Ginzburg-Landau functional. Calculus of Variations and Partial Differential Equations, 1(2):123-148, 1993. https://doi.org/10.1007/BF01191614.

[4] A. Contreras and X. Lamy. Biaxial escape in nematics at low temperature. Journal of Functional Analysis, 272(10):3987-3997, 2017. https://doi.org/10.1016/j.jfa.2017.01.012.

[5] T.A. Davis and E.C. Gartland, Jr. Finite element analysis of the Landaude Gennes minimization problem for liquid crystals. SIAM Journal on Numerical Analysis, 35(1):336-362, 1998. https://doi.org/10.1137/S0036142996297448.

[6] G. De Luca and A.D. Rey. Ringlike cores of cylindrically confined nematic point defects. The Journal of Chemical Physics, 126(9):904907, 2007. https://doi.org/10.1063/1.2711436.

[7] A. De Simone. Energy minimizers for large ferromagnetic bodies. Archive for Rational Mechanics and Analysis, 125(2):99-143, 1993. https://doi.org/10.1007/BF00376811.

[8] J.-I. Fukuda, H. Stark, M. Yoneyama and H. Yokoyama. Interaction between two spherical particles in a nematic liquid crystal. Physical Review E, 69(4):041706, 2004. https://doi.org/10.1103/PhysRevE.69.041706.

[9] E.C. Gartland, Jr. Scalings and limits of the Landau-de Gennes model for liquid crystals: A comment on some recent analytical papers. arXiv.org e-Print archive, 2015. Available from Internet: https://arxiv.org/abs/1512.08164v1.

[10] E.C. Gartland, Jr. and S. Mkaddem. Instability of radial hedgehog configurations in nematic liquid crystals under Landau-de Gennes free-energy models. Physical Review E, 59(1):563-567, 1999. https://doi.org/10.1103/PhysRevE.59.563.

[11] E.C. Gartland, Jr., P. Palffy-Muhoray and R.S. Varga. Numerical minimization of the Landau-deGennes free energy: Defects in cylindrical capillaries. Molecular Crystals and Liquid Crystals, 199(1):429-452, 1991. https://doi.org/10.1080/00268949108030952.

[12] D. Golovaty and J.A. Montero. On minimizers of a Landau-de Gennes energy functional on planar domains. Archive for Rational Mechanics and Analysis, 213(2):447-490, 2014. https://doi.org/10.1007/s00205-014-0731-3.

[13] D. Henao, A. Majumdar and A. Pisante. Uniaxial versus biaxial character of nematic equilibria in three dimensions. Calculus of Variations and Partial Differential Equations, 56(2):55, 2017. https://doi.org/10.1007/s00526-017-1142-8.

[14] S. Kralj, E.G. Virga and S. Žumer. Biaxial torus around nematic point defects. Physical Review E, 60(2):1858-1866, 1999. https://doi.org/10.1103/PhysRevE.60.1858.

[15] I.F. Lyuksyutov. Topological instability of singularities at small distances in nematics. Sov. Phys. JETP, 48(1):178-9, 1987. Translation of Zh. Éksp. Teor. Fiz. 75(1):358-360, 1978.

[16] A. Majumdar and A. Zarnescu. Landau-de Gennes theory of nematic liquid crystals: The Oseen-Frank limit and beyond. Archive for Rational Mechanics and Analysis, 196(1):227-280, 2010. https://doi.org/10.1007/s00205-009-02492 . 
[17] S. Mkaddem and E.C. Gartland, Jr. Fine structure of defects in radial nematic droplets. Physical Review E, 62(5):6694-6705, 2000. https://doi.org/10.1103/PhysRevE.62.6694.

[18] N.J. Mottram and C.J.P. Newton. Introduction to Q-tensor theory. arXiv.org e-Print archive, 2014. Available from Internet: https://arxiv.org/abs/1409. 3542 .

[19] L. Nguyen and A. Zarnescu. Refined approximation for minimizers of a Landaude Gennes energy functional. Calculus of Variations and Partial Differential Equations, 47(1-2):383-432, 2013. https://doi.org/10.1007/s00526-012-0522-3.

[20] P. Palffy-Muhoray, E.C. Gartland and J.R. Kelly. A new configurational transition in inhomogeneous nematics. Liquid Crystals, 16(4):713-718, 1994. https://doi.org/10.1080/02678299408036543.

[21] E. Pensenstadler and H.-R. Trebin. Fine structure of point defects and soliton decay in nematic liquid crystals. J. Phys. France, 50(9):1027-1040, 1989. https://doi.org/10.1051/jphys:019890050090102700.

[22] E.B. Priestley, P.J. Wojtowicz and P. Sheng. Introduction to Liquid Crystals. Plenum Press, New York, 1975.

[23] M. Ravnik and S. Žumer. Landau-deGennes modelling of nematic liquid crystal colloids. Liquid Crystals, 36(10-11):1201-1214, 2009. https://doi.org/10.1080/02678290903056095.

[24] R. Rosso and E.G. Virga. Metastable nematic hedgehogs. Journal of Physics A: Mathematical and General, 29(14):4247-4264, 1996.

[25] N. Schophol and T.J. Sluckin. Defect core structure in nematic liquid crystals. Physical Review Letters, 59(22):2582-2584, 1987. https://doi.org/10.1103/PhysRevLett.59.2582.

[26] A. Sonnet, A. Kilian and S. Hess. Alignment tensor versus director: Description of defects in nematic liquid crystals. Physical Review E, 52(1):718-722, 1995. https://doi.org/10.1103/PhysRevE.52.718.

[27] I.W. Stewart. The Static and Dynamic Continuum Theory of Liquid Crystals: A Mathematical Introduction. Taylor \& Francis, London, 2004.

[28] E.G. Virga. Variational Theories for Liquid Crystals. Chapman \& Hall, London, 1994.

[29] N.J. Walkington. Numerical approximation of nematic liquid crystal flows governed by the Ericksen-Leslie equations. ESAIM: Mathematical Modelling and Numerical Analysis, 45(3):523-540, 2011. https://doi.org/10.1051/m2an/2010065.

[30] P. Ziherl and S. Žumer. Fluctuations in confined liquid crystals above nematicisotropic phase transition temperature. Physical Review Letters, 78(4):682-685, 1997. https://doi.org/10.1103/PhysRevLett.78.682. 